\title{
Analysis of parameters influencing the jamming effect in circular braids
}

\author{
J. Ochola ${ }^{1,2}$, B. Malengier ${ }^{1}$, J. Githaiga $^{2}$ \& L. VanLangenhove ${ }^{1}$ \\ ${ }^{1}$ Department of Textiles, Ghent University, Belgium \\ ${ }^{2}$ Department of Industrial and Textile Engineering, \\ Moi University, Kenya
}

\begin{abstract}
Circular braids are tubular fabrics constructed using a set of helically winding yarns in a circular clockwise and counter-clockwise direction. Circular braided structures exhibit the behavior of rearranging on extension along the longitudinal axis. In this paper, a 3D model of a circular braided structure was developed using generic python, and rendered on $\mathrm{ABAQUS}{ }^{\circledR}$. A uniaxial tension was induced to the structure at different load values and the extension of the model analysed before and after the jamming state. The models were validated using experimental data and linear regression analysis to study the force-extension relationship during structural deformations in a braided structure. A linear regression model of the relationship between the peak force (y) and the tensile extension ( $\mathrm{x}_{1}$ was developed using linear regression at $95 \%$ confidence level. The multiple correlation coefficients (R) indicating a measure of the quality of the prediction of the dependent variable was established as 0.896 , for the model, this indicates a good level of prediction. The $\mathrm{R}^{2}$ value (also called the coefficient of determination) was 0.803 , this was the proportion of variance in the dependent variable that could be explained by the independent variables (technically, it is the proportion of variation accounted for by the regression model above and beyond the mean model). Therefore in this model the independent variables explained $80.3 \%$ of the variability of our dependent variable, maximum load (y). The extension $\left(\mathrm{x}_{1}\right)$ of the braided structure was found to have a significant impact on the model.
\end{abstract}

Keywords: circular, braid, regression, extension, tenacity. 


\section{Introduction}

A braid is a linear fibrous assembly composed of two main sets of interlacing yarns lying at a defined angle relative to the longitudinal axis of the structure. Braided structures can take a variety of forms such as: narrow fabric, circular fabric, tubular fabric, cord, and thread. Tubular braids, also referred to as "round" or "circular" braids are fibrous cylindrical shell of small diameter consisting of clockwise and anti-clockwise sets of mutually interlacing, spiraling yarns. The application of circular braided structures in engineering has been vast mainly in civil engineering, mechanical engineering, bio-medical, domestic goods among other uses. Nevertheless in all the applications where the circular braids have been used there is always the requirement for analysis of the mechanical performance of the structures to ascertain their suitability for the field of application. In this analysis theoretical models of the structures have been crucial. It is in these models where the geometrical aspects of the braids are developed to be used in the prediction of the performance of the structure during engineering analysis. In addition the theoretical modeling of the geometry of the braids can also be important in optimizing the manufacturing process of the structures. Studies on geometrical modeling of braided structures have been a subject of several research works over the years and have taken diverse approach. Early attempts on geometrical modeling of braided structures was performed for 2D structures. Brunnschweiler focused mainly on 2D modeling and analysis of braided structures [1]. He developed his theory on braided structures [2] by using the Pierce approach [3]. Later the studies developed into $3 \mathrm{D}$ domain and hence the modeling of braided structures shifted mostly to 3D structures. Zhang et al. [4] focused on the modeling of the yarn's actual motion in the circular braiding process, using a unique particle velocity approach. The approach was aimed at explaining the influence of the twist effect in the convergent zone on the braid angle decrease phenomena and the slowdown of the yarn deposition onto the mandrel in the presence of friction forces. Potluri et al. [5] while studying triaxial braiding, used 3D models to simulate the process with the need to optimize the parameters of the braids. Then Rawal and Potluri $[6,7]$ developed models for different shapes of braided structures by modeling yarn paths for different forms of braids and established that during braiding the path of the yarn on the mandrel is dependent upon the shape of the mandrel. The models implied that when the mandrel is circular then yarn path will be in the form of a helix. Wensuo et al. [8] undertook studies on using symmetry as a theoretical basis for deriving novel 3D braided geometry structures. Tuba Alpyildiz [9] performed 3D modeling of tubular structures, by developing the braid models by considering crimp, yarn paths, braid angle, number of yarns, yarn and mandrel diameter. Advancements of the topic on modeling braided structures resulted in analysis of the mechanical behaviour and structural performance of the braids. Mehemet [10] undertook a study on elasticity of potential tubular braided structures for medical applications using both experimentally and theoretically approach. The results established that the diameter of the braided tube and the number of filaments with its diameter have 
significant effect on the force measured from the braided tube. The models also showed that the diameter of the braided structures decreases with increase in length. Rawal et al. [11] studied the tensile properties of braided structures based on braid geometry, braid kinematics, and constituent monofilament properties, taking into account the changes in the braid geometry, including braid angle, diameter, and Poisson's ratio. They established that both the both braid angle and diameter are found to be critical design parameters to the model of jamming state of mono filaments. Bilisik [12] established that in jamming conditions, minimum jamming decreased the width of the unit cell structure but maximum jamming increased its width. The models also established that minimum jamming decreased the surface angle of the unit cell structure, while the maximum jamming increased the surface angle. Also, it was realized that jamming conditions influenced the density of the unit cell but did not affect the yarn length in the unit cell structures. Zheng [13], while studying the micro-structure of braids, established that the pitch length decreased with the braiding angle and numbers of yarns increased. Zhang et al. [14] performed a study on a 2D-braid model and established that the cover factor of a fabric braided on a particular braider depends on three variables: braid angle, helical length, and braid diameter. They further reported that the cover factor of a braided structure is a function of braid angle and diameter and maintains a constant helical length between its tensile and compressive jammed states. According to Zhang et al. [14] a stable jammed slate with maximum crimp is found to exist when the braid angle is $45^{\circ}$ and the helical length is a minimum. When the braid diameter is held constant by braiding on a constant-diameter mandrel, the cover factor is increased by decreasing the helical length or increasing the braid angle. Hence they concluded that the cover factor is directly related to the fabric width as a single independent variable. When the yarn cannot be considered as a flat strip but must instead be considered to have a circular cross-section [14]. Most of these studies and many others successfully developed models for braided structures and made attempts to explain the structural deformation in circular braids. However, they did not take into account the influence of mechanical parameters such as uniaxial force and tensile extension in inducing structural changes in the circular braids such as jamming of the constituent elements within the structure. The uniaxial force in the braided structures plays an important role in initiating the displacement of yarns in a braid, thus causing jamming to occur. Ideally, the significance of the tensile forces is to cause deformations within the braid by causing changes to the radius and the length of the structure as a result of the movement of the yarns, thus extending the structure. Mechanical properties of the braided structure should therefore be optimized to ensure a braided structure would withstand high forces at minimal deformation. The possibility of a braid possessing unbalanced force-extension properties would be catastrophic during application in conditions where stability of the structure is required. Due to the problems associated with the deformation of the circular braid under constant loading and unloading during application, it would be appropriate therefore to establish models for the relationship between the force and extension for the structure. The purpose of this research is to use regression 
and 3D modeling techniques to investigate the statistical significance and relationship between the applied force and extension in a circular braid.

\section{Material and experimental methods}

The samples used for testing were made of PolyPropylene filament yarns, 5 samples of circular braids were selected for the test, the data of which is shown in Table 1. The samples were tested using Instron tensile testing equipment shown in Figure 1.

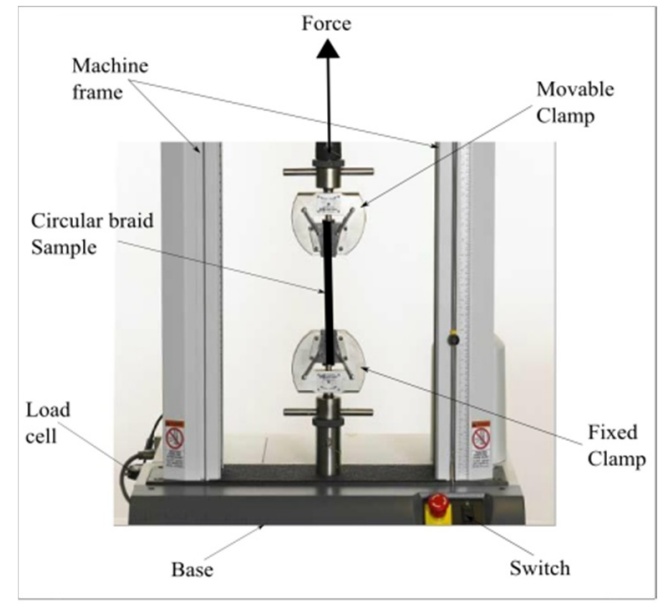

Figure 1: Experimental setup showing components of Instron tensile tester used for cyclic testing of circular braided structure samples.

Table 1: $\quad$ Test data from uniaxial tensile tests.

\begin{tabular}{|l|c|c|c|}
\hline Force, F/[N] & Minimum & Maximum & Average \\
\hline Sample 1, $\mathrm{F}_{1}$ & 0.07 & 268.68 & 33.31 \\
\hline Sample 2, $\mathrm{F}_{2}$ & 0.39 & 287.22 & 36.88 \\
\hline Sample 3, $\mathrm{F}_{3}$ & 0.49 & 218.52 & 23.42 \\
\hline Sample 4, $\mathrm{F}_{4}$ & 0.35 & 236.7 & 27.48 \\
\hline Sample 5, F5 & 0.50 & 248.91 & 29.26 \\
\hline \multicolumn{4}{|l|}{} \\
\hline Extension, e/[mm] & Minimum & Maximum & Average \\
\hline Sample 1, e 1 & 0.02 & 9.998 & 5.03 \\
\hline Sample 2, e 2 & 0.27 & 9.996 & 4.90 \\
\hline Sample 3, e 3 & 0.23 & 9.996 & 4.91 \\
\hline Sample 4, e 4 & 0.24 & 9.996 & 4.91 \\
\hline Sample 5, e 5 & 0.26 & 9.996 & 4.90 \\
\hline
\end{tabular}


Cyclic testing was adopted for the experiments with a ramp of $10 \mathrm{~mm}$ at the rate of $100 \mathrm{~mm} / \mathrm{min}$; the load cell used was $500 \mathrm{~N}$ at a preload of $0.5 \mathrm{~N}$. The minimum, maximum and average uniaxial force and extension was recorded in Table 1 for 100 peaks of the ramp extension of $10 \mathrm{~mm}$.

\section{Results and discussion}

Linear regression was used to predict the value of a uniaxial force, $F(y)$ in a circular braid based on the value of its tensile extension, $e\left(x_{1}\right)$. The force $(y)$ is considered therefore as the dependent. The extension $\left(\mathrm{x}_{1}\right)$ was used in predicting the force on the structure during deformation and hence was considered to be the independent variable. The regression analysis was performed using SPSS $22^{\circledR}$. The analysis was then used to determine the overall fit of the model and the relative contribution of the predictor to the total variance that the model could be able to explain.

\subsection{Statistical significance}

The F-ratio in the ANOVA Table 2 was used to test whether the overall regression model is a good fit for the data. The results in the table indicates that the independent variable is statistically significant in predict the dependent variable at $p<0.0005$. This implies that the regression model is a good fit of the data.

Table 2: $\quad$ Analysis of variance.

\begin{tabular}{|l|c|c|c|c|c|}
\hline & $\boldsymbol{d} \boldsymbol{f}$ & $\boldsymbol{S S}$ & $\boldsymbol{M S}$ & $\boldsymbol{F}$ & Significance $\boldsymbol{F}$ \\
\hline Regression & 1 & 1548835.34 & 1548835.34 & 1305.315647 & $5.8921 \mathrm{E}-115$ \\
\hline Residual & 320 & 379699.2014 & 1186.560004 & & \\
\hline Total & 321 & 1928534.542 & & & \\
\hline
\end{tabular}

\subsection{Model coefficients}

The regression coefficients in Table 3 would indicate how much the dependent variable $\left(\mathrm{x}_{1}\right)$ varies with an independent variable $(\mathrm{y})$. The coefficient for $\mathrm{x}_{1}$ is equal to 23.32. This implies for a circular braid under uniaxial force would increase by $23.32 \mathrm{~N}$, for every increase in the tensile extension.

Table 3: Regression coefficients.

\begin{tabular}{|c|c|c|c|c|c|}
\hline & Coefficients & $\begin{array}{c}\text { Standard } \\
\text { Error }\end{array}$ & P-value & Lower 95\% & Upper 95\% \\
\hline Intercept & -52.42 & 3.73 & $2.8 \mathrm{E}-35$ & -59.76 & -45.09 \\
\hline $\mathrm{e} /[\mathrm{mm}]$ & 23.32 & 0.65 & $5.89 \mathrm{E}-115$ & 22.05 & 24.59 \\
\hline
\end{tabular}

In determining how well the regression model fits the data from the model summary results it was established that the model had 0.896 as the multiple correlation coefficient $(\mathrm{R})$ which can be considered to be a measure of the 
quality of the prediction of the dependent variable; ultimate load (y). This indicates a good level of prediction. The model produced an $\mathrm{R}^{2}$ value of 0.803 ; this was measure of the proportion of variation accounted for by the regression model and beyond the mean model. There from the $\mathrm{R}^{2}$ value of 0.803 , it implies that the independent variable could explain $80.3 \%$ of the variability of our dependent variable, uniaxial force.

The statistical significance of each of the independent variable was also tested to establish whether the coefficients are equal to 0 (zero) in the population. It was established that the tensile extension $\left(\mathrm{x}_{1}\right)$ had realistic significance to the model, both with a $\mathrm{p}<0.005$, this could also be established in Table 3 .

The predicted value of the uniaxial force indicated a linear relationship with the tensile extension as shown in Figure 2, which indicates that the is a direct influence of changes in the extension of the structure with the applied force.

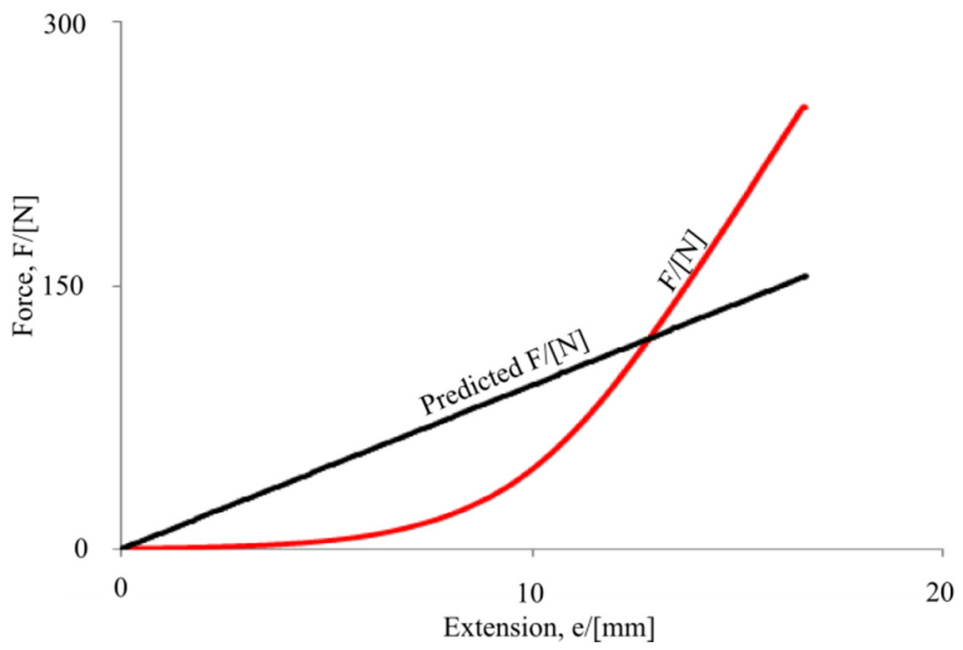

Figure 2: The predicted value of the uniaxial force plotted against the tensile extension compared to the curve of the experimental force versus extension. The curve shows a uniform linear relationship between the extension and the uniaxial force of the circular braided structure.

\subsection{Modeling of structural deformations in the circular braid}

The structural deformations of the circular braid were modeled using generic Python programming and ABAQUS ${ }^{\circledR}$ to establish the profile of the force $(\mathrm{F})$ and the extension (e), during uniaxial testing of the model. The 3D model of a braided structure was developed as shown in Figure 3. The model was developed by plotting the helical coordinates derived by equation 1 for the yarn path in clockwise and anticlockwise directions, using python scripts. The coordinates in the $y$-axis becomes negative in the anticlockwise direction. 


$$
\left\{\begin{array}{l}
x=R \cos \theta \\
y= \pm R \sin \theta \\
z=R \theta(\tan \alpha)
\end{array}\right.
$$

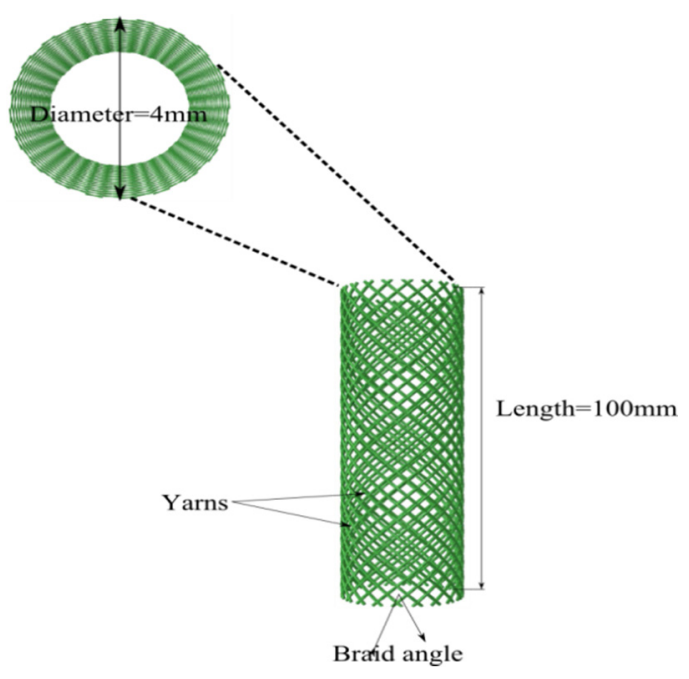

Figure 3: The 3D model of a circular braided structure developed using generic python script, showing the structural components of a circular braid including the interlacing of yarns and the braid angle between the yarns.

The input file from the script was imported and rendered on ABAQUS. The experimental cyclic testing parameters were replicated in the model as shown by simulation images in Figure 4 and the results plotted and compared to those of experimental data as shown in Figure 5.

The simulation of the longitudinal displacement of helical yarns in the $3 \mathrm{D}$ braid structure shown in Figure 4 was performed for $20 \mathrm{~mm}$ extension of the braided.

The structure showed significant changes along its radius and length. In order to study the extent of the structural changes in structure, a plot was developed for the ultimate force and extension; the plot was compared to a curve from the experimental data within similar range, the curves shown in Figure 5 suggests that the structure would begin to yield after an extension of $10 \mathrm{~mm}$, in both theoretical and experimental data. After this extension, the rate of change of the extension of the curves with force is higher than the preceding regions of the curves. The curves therefore indicate that though the extension of the structure would increase with an increase in the force, as seen earlier by the regression model, it now also evident that the rate of increase of the extension 


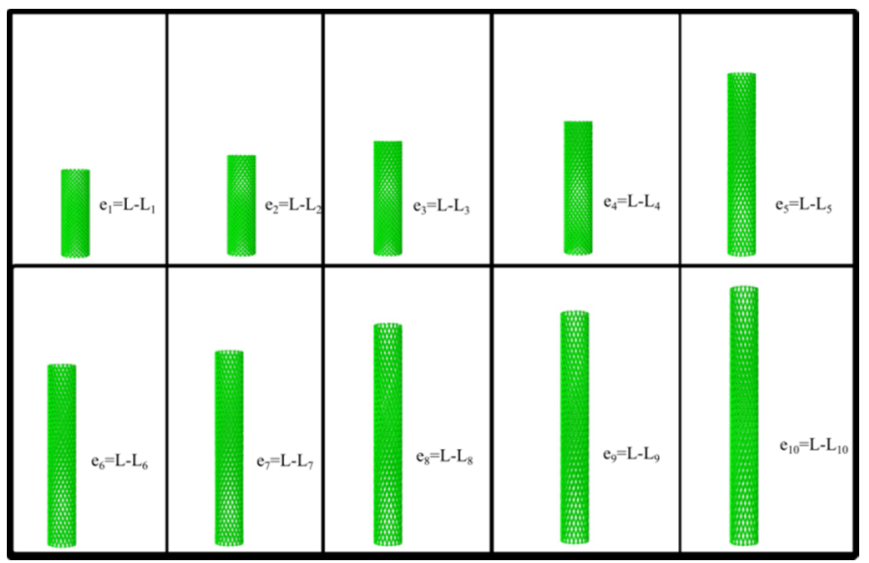

Figure 4: Simulation images indicating the structural changes of the circular braid under uniaxial force, showing the change in length of the structure.

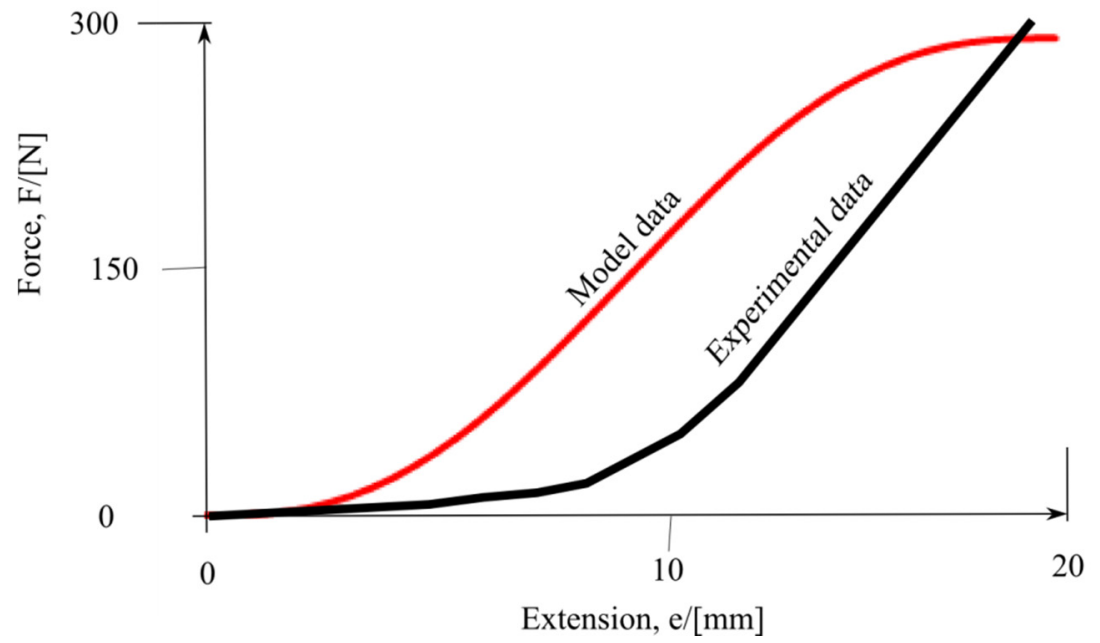

Figure 5: The model data curve and experimental data curve showing comparison between the modelled and experimental profile of the braided structure due to deformations.

and force in the structure would be higher after some point and in this case after $10 \mathrm{~mm}$. This could be evidence of the point at which the helical angle between the yarns is reduced while the yarns gets closer to each other as the displacement of the yarns occur. At this point jamming of the yarns is believed to begin, because the angle between the yarns becomes too small hence no further angular 
displacement is allowed and further application of the force results in stretching of the constituent yarns within the braided structure.

\section{Conclusions}

In modeling the force and extension relationship in a circular braided structure using linear regression technique it was established that the extension of the structure would have a statistical significant positive effect on corresponding force applied in the structure. It was evident from the model that the force in structure would increase as the extension of the structure increases. However, further studies of the phenomena using 3D models indicated that the rate of change between the curves for the force and extension would increase when the yarns within the structure come very close to each other and their angular displacement becomes minimal. This phenomenon describes a point during the uniaxial loading in which the yarns within the structure reach the jamming point and further extension of the structure would leading to a decrease in the rate of load as the structure starts deforming.

\section{References}

[1] Brunnschweiler D. Braids and Braiding. Journal of the Textile Institute 1953; 44:666.

[2] Brunnschweiler D. The Structure and Tensile Properties of Braids. Journal of the Textile Institute 1954; 45.

[3] Pierce F. T. The Geometry of Cloth Structure. Journal of the Textile Institute $1937 ; 28$.

[4] Zhang Q., Beale D., Broughton R. Analysis of Circular Braiding Process, Part 1: Theoretical Investigation of Kinematics of the Circular Braiding Process. Journal of Manufacturing Science and Engineering 1999; 121:345.

[5] Potluri P., Rawal A., Rivaldi M., Porat I. Geometrical modelling and control of a triaxial braiding machine for producing $3 D$ preforms. Composites 2003; 34:481-492.

[6] Rawal A. and Potluri P. Geometrical Modeling of the Yarn Paths in Threedimensional Braided Structures. Journal of Industrial Textiles 2005; 35:2.

[7] Rawal A. and Potluri P. Prediction of Yarn Paths in Braided Structures Formed on a Square. Journal of Industrial Textiles 2007; 36.

[8] Wensuo M., Bingqian Y. and Xiaozhong R. Derivation of $3 D$ Braided Geometry Structures from Braided Symmetry Group. 2011; 5:28-34.

[9] Tuba A. 3D tubular braided structures performed. Textile Research Journal 2012; 82.

[10] Mehemet Y. Analysis of Elastic Deformation of Braided Tubular Structures for Medical Applications. Journal of Engineering Sciences 2001; 7:277-285.

[11] Rawal A., Kumar R. and Saraswat H. Tensile mechanics of braided sutures. Textile Research Journal 2012; 82:16. 
102 High Performance and Optimum Design of Structures and Materials II

[12] Bilisik K. Three-dimensional axial braided preforms: experimental determination of effects of structure-process parameters on unit cell. Textile Research Journal 2011; 81:2095.

[13] Zheng X. Microstructure Analysis of 4-Step Three-Dimensional Braided Composite. Chinese Journal of Aeronautics 2003; 16:3.

[14] Zhang Q., Beale D., Adanur S., Broughton R. M. and Walker R. P. Structural Analysis of a two dimensional Braided Fabric. Journal of the Textile Institute 1997; 88:41-5. 